\title{
Evaluation of novel cyclic analogues of apelin
}

\author{
JURI HAMADA $^{1,3}$, JUNKO KIMURA ${ }^{1}$, JUNJI ISHIDA ${ }^{2}$, TAKEO KOHDA ${ }^{1}$, SETSUO MORISHITA $^{1}$, \\ SHIGEYASU ICHIHARA ${ }^{1}$ and AKIYOSHI FUKAMIZU ${ }^{1-3}$ \\ ${ }^{1}$ Ankhs Incorporated, Tsukuba Industrial Liaison and Cooperative Research Center, ${ }^{2}$ Graduate School of Life \\ and Environmental Sciences, ${ }^{3}$ Center for Tsukuba Advanced Research Alliance (TARA), \\ University of Tsukuba, Tsukuba, Ibaraki 305-8577, Japan
}

Received April 23, 2008; Accepted June 17, 2008

DOI: 10.3892/ijmm_00000054

\begin{abstract}
Apelin regulates various cell signaling processes through interaction with its specific cell-surface receptor, APJ, which is a member of a seven transmembrane $G$ proteincoupled receptor superfamily. To develop a novel apelin analogue, we synthesized cyclic analogues of minimal apelin fragment RPRLSHKGPMPF (apelin-12), and evaluated their bioactivities in a recombinant human APJ-expressed cell line. Three cyclic analogues were synthesized: cyclo apelin-12 (C1) in combination with amino-terminal to carboxy-terminal, cyclourea apelin-12 (C3) in combination with aminoterminal and amino acid side chain at positions 7 , and cyclic apelin-12 (C4) in combination with amino acid side chain at positions 7 to carboxy-terminal. All cyclic analogues exhibited dose-dependent inhibitory effects against forskolin-induced cyclic adenosine monophosphate (cAMP) accumulation, and the maximal effects were almost abolished by pertussis toxin (PTx) treatment. Moreover, they could modulate the intracellular signaling pathways composed of Akt and extracellular signal-regulated kinase 1/2 (ERK1/2) serine/threonine protein kinases in PTx-sensitive manner. This is the first approach to apply cyclization on apelin, and these results provide the basis for the development of drug-like apelin analogues.
\end{abstract}

\section{Introduction}

Apelin is an endogenous ligand peptide of APJ (a putative receptor protein related to the angiotensin-type 1 receptor, AT1) (1), which is a member of a seven transmembrane G protein-coupled receptor superfamily. Preproapelin synthesized as a 77 amino acid polypeptide is cleaved to multiple short forms of different sizes. Peptides of 12, 13, 17 and 36 amino acids in length including $\mathrm{C}$-terminal portion are known to

Correspondence to: Dr Akiyoshi Fukamizu, Center for Tsukuba Advanced Research Alliance, Graduate School of Life and Environmental Sciences, University of Tsukuba, Tsukuba, Ibaraki 305-8577, Japan

E-mail: akif2@tara.tsukuba.ac.jp

Key words: apelin, G protein be biologically active $(2,3)$. The APJ has a $31 \%$ amino acid sequence homology with the AT1, but does not display specific binding to angiotensin II, which is the ligand of AT1 and plays a central role in blood pressure and water and electrolyte homeostasis. Both apelin and APJ are expressed in a wide range of tissues, such as the cardiovascular and the central nervous system $(4,5)$. Exogenous apelin administration alters cardiovascular function, blood pressure, body temperature, body fluid and behaviors involved in food intake and water intake $(4,5)$. In addition, APJ was also identified as a human immunodeficiency virus type I (HIV-1) coreceptor, and apelin was shown to block HIV-1 entry through APJ $(3,6)$.

Currently, there are growing lines of evidence obtained from clinical studies that several human cardiovascular diseases are accompanied by changes in apelin and/or APJ expression in cardiovascular tissues (5). In animal studies, it was shown that apelin exerts vasodilation and positive inotropic actions ex vivo and in vivo (7-10). Apelin activates endothelial nitric oxide synthase (eNOS) and consequently stimulates nitric oxide (NO) release from the vascular endothelial cells, indicating the possible protective effect of apelin-APJ system on cardiovascular diseases through NO-dependent mechanism (9). In addition, the administration of apelin also ameliorated prognosis for heart function under pathological condition, such as experimental ischemia and reperfusion injury (11-14). Thus, it has been implicated that apelin plays an important role in the maintenance of cardiovascular homeostasis, and APJ has a potential to be a novel therapeutic target of cardiovascular dysfunction $(4,5)$. However, there are no available chemical small compounds for APJ.

The cyclization can be applied to a variety of peptides to improve their agonist or antagonist potency, including bioactivity, selectivity and bioavailability (15). We therefore made an attempt to synthesize cyclic apelin analogues based on apelin-12 fragment, which was identified as a minimum unit ligand for APJ, and evaluated their bioactivities in cell-based assays. APJ was demonstrated to exhibit apelindependent inhibition of adenylyl cyclase via PTx-sensitive $\mathrm{G}_{\mathrm{i} / \mathrm{o}}$ proteins, when expressed in recombinant cell lines (16-18). Moreover, in these cells, it was also shown that apelin induced the activation of the PI3K/Akt and MEK1/ERK1/2 signaling pathways. In the present study, we validated the potency of cyclic analogues of apelin by using these cellular signal indicators in vitro. 


\section{Materials and methods}

Peptides and reagents. Peptides were synthesized by Peptide Institute, Inc. (Osaka, Japan). The sequence data of synthetic peptide are shown below: pE-apelin-13: pGlu-Arg-Pro-ArgLeu-Ser-His-Lys-Gly-Pro-Met-Pro-Phe; cyclo apelin-12 (1-12): cyclo [Arg-Pro-Arg-Leu-Ser-His-Lys-Gly-Pro-Met-Pro-Phe] (1-12); cyclourea apelin-12 (1-7): cyclourea [Arg-Pro-ArgLeu-Ser-His-Lys-Gly-Pro-Met-Pro-Phe] (1-7); cyclo apelin-12 (7-12): cyclo [Arg-Pro-Arg-Leu-Ser-His-Lys-GlyPro-Met-Pro-Phe] (7-12). The characters used for the amino acids are the standard IUPAC. 'pE' and 'pGlu' represent a pyroglutamic acid residue. Cyclo apelin-12 (1-12), cyclourea apelin-12 (1-7) and cyclo apelin-12 (7-12) are referred to simply as $\mathrm{C} 1, \mathrm{C} 3$ and $\mathrm{C} 4$, respectively. The number in the brackets indicates positions of amino acids of which terminalgroup and side chain are covalently bound. A high performance liquid chromatography analysis of synthetic peptides exhibited a single peak on an analytical column with a homogeneity of $>97 \%$. Hydrolyzed peptides at $1 \mathrm{mM}$ concentration in water were stored at $-20^{\circ} \mathrm{C}$ before use. Forskolin was purchased from Sigma-Aldrich (St. Louis, MO, USA). Ro-20-1724 [4-(butoxy4-methoxybenzyl)-2-imidazolidinone] was from Biomol Research Laboratories, Inc. (Plymouth Meeting, PA, USA). PTx was from Seikagaku Corp. (Tokyo, Japan). The primary antibodies were purchased from as follows: mouse monoclonal anti-ß-actin from Sigma-Aldrich; mouse monoclonal antiphosphoAkt (Ser473), rabbit polyclonal anti-Akt, and rabbit polyclonal anti-phosphoERK1/2 (Thr202/Tyr204) from Cell Signaling Technology, Inc. (Danvers, MA, USA); mouse monoclonal anti-ERK2 from Upstate Biotechnology (Lake Placid, NY, USA). A rabbit polyclonal anti-APJ antibody was raised against a GST-mouse APJ (amino acid residues 311-377) fusion protein expressed in bacteria and injected into rabbits. Serum from immunized rabbits was affinity purified using the antigen (ProteinPurify Co., Ltd., Gunma, Japan). The horseradish peroxidase-conjugated secondary antibodies were purchased from GE Healthcare (Buckinghamshire, $\mathrm{UK})$.

Establishment of stable cell lines. HEK293-T cells were grown in growth medium: Dulbecco's modified Eagle's medium (DMEM) supplemented with $10 \%$ heat-inactivated fetal bovine serum (FBS), non-essential amino acids, $100 \mathrm{U} / \mathrm{ml}$ penicillin and $100 \mu \mathrm{g} / \mathrm{ml}$ streptomycin at $37^{\circ} \mathrm{C}\left(5 \% \mathrm{CO}_{2}\right.$ and $95 \%$ humidity). Cells were transfected with the coding region of the human APJ gene expressed pcDNA3.1/Zeo(+) (Invitrogen) using TransFast (Promega). Stable transformants (so-called APJ/293-T) were selected and propagated with $200 \mathrm{ng} / \mathrm{ml}$ zeocin (Invitrogen). To confirm the protein expression of $A P J$, cells were solubilized in Laemmli's sample buffer and subjected to immunoblot analysis (see below). HEK293-T cells stably transfected with pcDNA3.1/Zeo(+) (so-called mock/293-T) were used to verify non-specific action of synthetic analogues.

cAMP assay. Cells were plated at 20,000 cells per well into type I collagen-coated 96-multiwell plates and allowed to adhere overnight in $90 \mu \mathrm{l}$ of growth medium supplemented with $20 \mathrm{mM}$ HEPES buffer at $37^{\circ} \mathrm{C}\left(5 \% \mathrm{CO}_{2}\right.$ and $95 \%$ humidity). For PTx treatment, cells were incubated with $100 \mathrm{ng} / \mathrm{ml}$ of PTx for 12-15 h before assay. On the day of assay, the cell cultured plates were equilibrated at room temperature for $20 \mathrm{~min}$ and then $5 \mu 1$ of aliquots of $1.95 \mathrm{mM}$ Ro-20-1724 were added to the cultured medium at final concentration of $100 \mu \mathrm{M}$ and incubated at room temperature for another $20 \mathrm{~min}$. After incubation, $5 \mu \mathrm{l}$ of aliquots of different doses of peptide analogues containing $0.1 \mathrm{mM}$ forskolin were loaded $5 \%$ of the final volume (forskolin; at final concentration of $5 \mu \mathrm{M}$ ) and incubated at room temperature for $10 \mathrm{~min}$. After stimulation, the reaction was stopped by adding of 5-fold diluted lysis buffer 1A, a component of the cAMP enzymeimmunoassay (EIA) system (Amersham Biosciences), and then, $100 \mu \mathrm{l}$ aliquots of cell lysate served as cAMP measurement according to the manufacturer's instructions. The sample absorbance at $450 \mathrm{~nm}$ was measured on Wallac $\mathrm{ARVO}_{\mathrm{SX}}$ Multilabel Counter (Perkin-Elmer, Tokyo, Japan) and analyzed using WorkOut Ver. 1.5 software (Perkin-Elmer).

Immunoblot analysis. Cells were plated at 150,000 cells per well into poly-D-lysine-coated 24-multiwell plates and allowed to adhere overnight in $500 \mu 1$ of growth medium at $37^{\circ} \mathrm{C}\left(5 \% \mathrm{CO}_{2}\right.$ and $95 \%$ humidity). In the next assay, the cultured medium was replaced to $495 \mu 1$ of serum-free DMEM and the cells were further incubated for $24 \mathrm{~h}$. For PTx treatment, cells were incubated with $100 \mathrm{ng} / \mathrm{ml}$ of PTx for $12 \mathrm{~h}$ before assay. On the day of assay, $5 \mu \mathrm{l}$ aliquots of peptide analogues were added to the cultured medium and incubated at $37^{\circ} \mathrm{C}$. After stimulation, the plates were left to stand in ice water for a few min, and then, the medium was removed. The cells were washed with ice-cold PBS, followed by immediate solubilization with $100 \mu 1$ of Laemmli's sample buffer containing $100 \mathrm{mM}$ dithiothreitol. After sonication and treatment at $99^{\circ} \mathrm{C}$ for $3 \mathrm{~min}$, the cell lysates were centrifuged at $4^{\circ} \mathrm{C}$ for $5 \mathrm{~min}$ and the super-natants were subjected to SDS-PAGE (10\% polyacrylamide) and transferred to a nitrocellulose membrane (Protoran; Schleicher\&Schuell, Dassel, Germany). Filters were blocked with skim milk and then blotted with primary antibodies. After incubation, blots were incubated with the corresponding secondary antibodies, followed by visualization via chemiluminescent detection using Immobilon Western HRP Substrate (Millipore Corp., Billerica, MA, USA), and data images were captured with LAS-3000 (Fujifilm, Tokyo, Japan).

Data analysis. Duplicates or triplicates were performed and at least three independent trials with similar results were done for each set of experiments. Non-linear regression analysis of dose-response data was performed using Prism4 (GraphPAD Software, San Diego, CA, USA). One-way ANOVA and Bonferroni's multiple comparison test with 95\% confidence were adapted for the evaluation of the significance of the differences between groups. The data are presented as means \pm standard error (SE) of at least three independent experiments.

\section{Results}

We synthesized three cyclic apelin analogues using apelin-12 as a template (Fig. 1). The structure of synthetic peptides 
pE-apelin-13 pGlu1-Arg-Pro-Arg-Leu-Ser-His-Lys-Gly-Pro-Met-Pro-Phe

apelin-12 Arg-Pro-Arg-Leu-Ser-His-Lys-Gly-Pro-Met-Pro-Phe

\begin{tabular}{ll|} 
C1 Arg-Pro-Arg-Leu-Ser-His-Lys-Gly-Pro-Met-Pro-Phe \\
C3 \\
C4 Co \\
Arg-Pro-Arg-Leu-Ser-His-Lys-Gly-Pro-Met-Pro-Phe
\end{tabular}

Figure 1. Structure of synthesized apelin and cyclic analogues. The combined amino acids in cyclic analogues are represented by line in the alignment of the sequences of apelin-12.

A

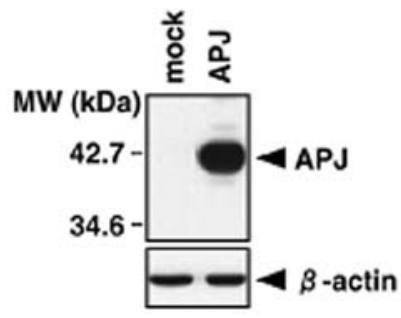

B

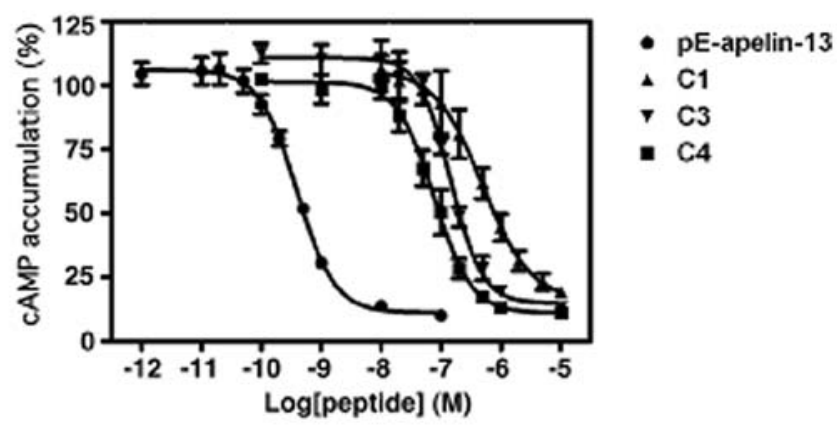

C
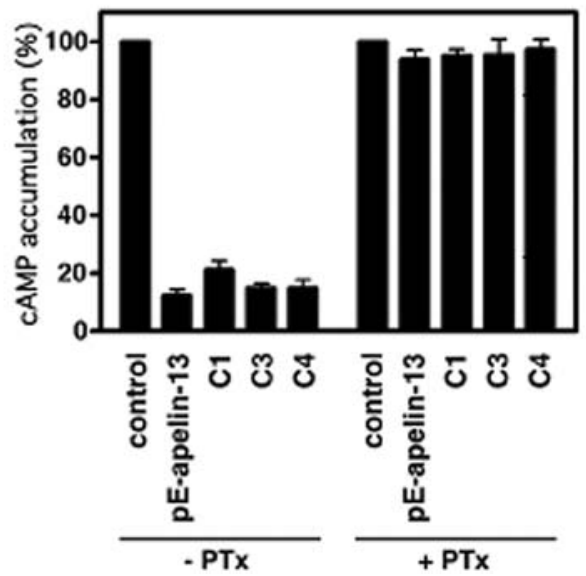

D

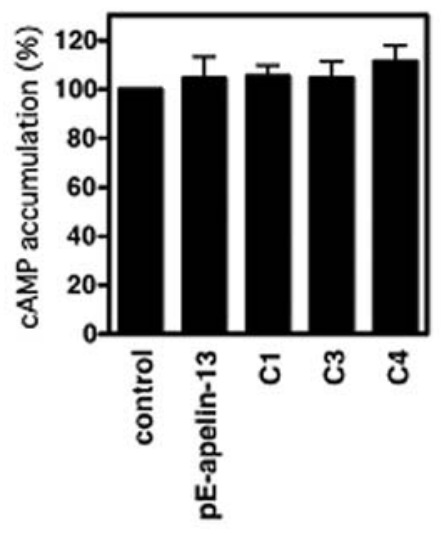

Figure 2. Inhibitory activities of apelin and cyclic analogues for cAMP accumulation. (A) APJ stable expression in APJ/293-T cells was determined by immunoblotting using anti-APJ antibody. Upper panel, immunoreactive band of APJ is detected in APJ/293-T (right lane) but not in mock/293-T (left lane) cells. Bottom panel, immunoreactive band of B-actin in APJ/293-T (right lane) is comparable to that observed in mock/293-T (left lane) cells. Molecular weight (MW) markers are indicated in kilodaltons $(\mathrm{kDa})$ on the left of panel. (B) Dose-response curves of forskolin-induced cAMP accumulation in APJ/293-T cells for apelin and cyclic analogues. (C) Effects of apelin (100 nM) and cyclic analogues $(10 \mu \mathrm{M})$ on cAMP accumulation in APJ/293-T cells were almost abolished by PTx treatment. (D) No significant effects of apelin (100 nM) and cyclic analogues $(10 \mu \mathrm{M})$ on cAMP accumulation were observed in mock/293-T cells. The $\mathrm{X}$ axis is the negative logarithm of peptide concentration and the Y axis is the \% of cAMP accumulation of forskolin control. Values are expressed as means $\pm \mathrm{SE}$ of at least three independent experiments.

were followed: cyclo apelin-12 (C1) in combination with amino-terminal to carboxy-terminal, cyclourea apelin-12 (C3) in combination with amino-terminal and amino acid side chain at positions 7, and cyclic apelin-12 (C4) in combination with amino acid side chain at positions 7 to carboxy-terminal. The amino-terminal pyroglutamylated apelin-13 (pE-apelin-13) was also synthesized for use as a reference drug for cell-based assays.
To evaluate the bioactivities of the synthetic analogues in cell-based assays, we established recombinant cell lines and assessed a specific stable expression of APJ in HEK293-T cell trasnsfected with human $A P J$ gene. Immunoreactive band of the APJ $(\sim 42 \mathrm{kDa})$ was predominantly detected in the APJ/293-T cell line by immunoblotting using an anti-APJ antibody, while not in the mock/293-T cell line (Fig. 2A). First, we performed the cAMP assay using the APJ/293-T 
Table I. Activity profile of synthetic peptides from inhibitory effects on cAMP accumulation.

\begin{tabular}{lccc}
\hline Peptide & $\mathrm{EC}_{50}(\mathrm{nM})$ & $\begin{array}{c}\text { Relative } \mathrm{EC}_{50} \\
\text { value }\end{array}$ & $\begin{array}{c}\text { Maximal } \\
\text { inhibition (\%) }\end{array}$ \\
\hline pE-apelin-13 & $0.38 \pm 0.04$ & 1 & $10.0 \pm 1.2$ \\
C1 & $493.17 \pm 88.76$ & 1275 & $19.3 \pm 2.1$ \\
C3 & $144.27 \pm 5.25$ & 373 & $13.1 \pm 2.0$ \\
C4 & $78.62 \pm 14.88$ & 203 & $11.3 \pm 1.3$ \\
\hline
\end{tabular}

cell line, when exposed to different doses of peptide analogues, and determined the relative potency (Fig. 2B and Table I). All synthetic peptides showed dose-dependent inhibitory effects on forskolin-induced cAMP accumulation in the APJ/293-T cells. The effective concentration ranges of every cyclic analogue shifted to higher concentration than that of pE-apelin. The $\mathrm{EC}_{50}$ (median effective concentration) value of pE-apelin-13 was $0.38 \pm 0.04 \mathrm{nM}$, in quantitative agreement with previous reports (19), yielding the maximal inhibition of $\sim 10 \%$ at $100 \mathrm{nM}$. The $\mathrm{EC}_{50}$ value of $\mathrm{C} 1$ was $493.17 \pm 88.76 \mathrm{nM}$ (relative 1,275-fold increase to pE-apelin-13), yielding the maximal inhibition of $\sim 20 \%$ at $10 \mu \mathrm{M}$. The $\mathrm{EC}_{50}$ values of C3 and C4 were $144.27 \pm 5.25 \mathrm{nM}$ (relative 373-fold increase to pE-apelin-13) and 78.62 $\pm 14.88 \mathrm{nM}$ (relative 203-fold increase to pE-apelin-13), respectively, yielding the similar maximal inhibition of $\sim 10 \%$ at $10 \mu \mathrm{M}$ in an experimental range of concentration of peptides. To determine whether the effects of pE-apelin-13 and cyclic analogues were specific for APJ, we subsequently investigated the PTx-sensitivity and the non-specific action in the mock/293-T cells. The inhibitory effects of pE-apelin-13 (at $100 \mathrm{nM}$ ) and three cyclic analogues (at $10 \mu \mathrm{M}$, each) on forskolin-induced cAMP accumulation were almost abolished in APJ/293-T cells with PTx pretreatment (Fig. 2C). No significant inhibitory effects of any peptides, at the same concentration as mentioned above, were observed in the mock/293-T cells (Fig. 2D). Taken together, we showed that all cyclic analogues were APJ agonists.

Secondly, to assess whether cyclic analogues could modulate the intracellular signaling pathways, we examined the activation of Akt and ERK1/2 molecules by the immunoblot analysis using antibodies against phosphorylated Akt (Ser473) and ERK1/2 (Thr202/Tyr204), which are known to reflect its activation. In the APJ/293-T cells, the phosphorylation levels of both Akt and ERK1/2 were rapidly increased within 2 min after exposure to pE-apelin-13 (at $1 \mu \mathrm{M}$ ) (Fig. 3A). The enhanced phosphorylation level of Akt was sustained during the experimental period (0-30 min), whereas that of ERK1/2, by contrast, was transient during the short period (2-5 $\mathrm{min}$ ) and subsequently depressed close to the basal level after $15 \mathrm{~min}$. Exposure to the cyclic analogues (at $1 \mu \mathrm{M}$ ) also rapidly induced both Akt and ERK1/2 phosphorylation and showed similar time-course manner to pE-apelin-13. Though a slight increment of the phosphorylation levels of ERK1/2 compared with exposure to pE-apelin-13, all cyclic analogues increased the phosphorylation levels of Akt to a similar extent compared with pE-apelin-13 after exposure. We also tested the dosedependent effects of pE-apelin-13 and cyclic analogues on the activation of these molecules. The APJ/293-T cells were exposed to different doses of peptides in a concentration range from $1 \mathrm{nM}$ to $1 \mu \mathrm{M}$ for $5 \mathrm{~min}$, followed by immunoblot analysis. As shown in Fig. 3B, the phosphorylation level

A

vehicle

apelin

C1

C3

C4

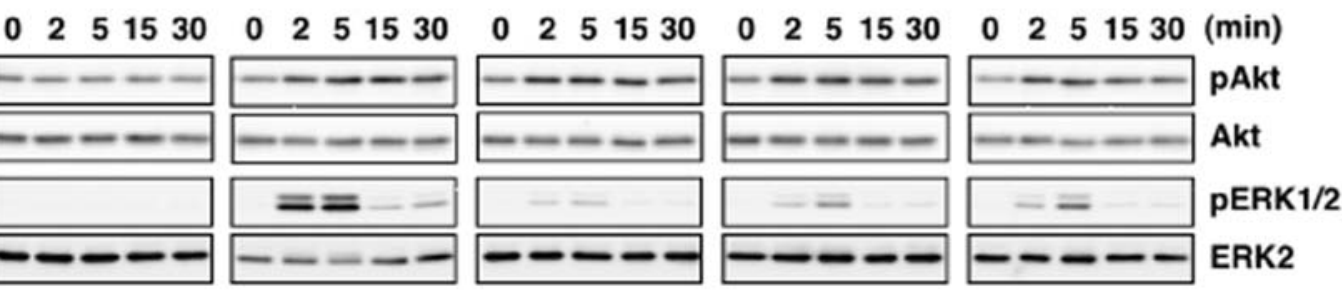

B
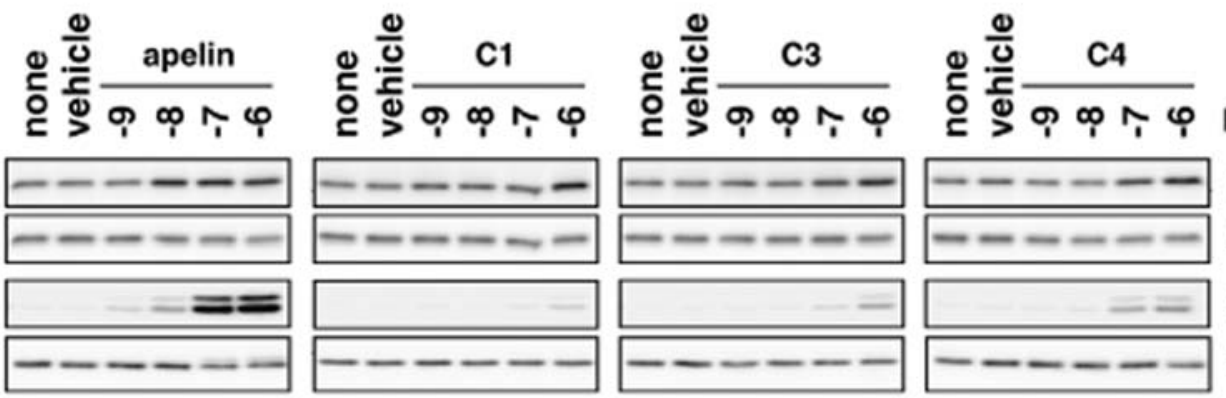

Log[peptide] (M)

pAkt

Akt

pERK1/2 ERK2

Figure 3. Time- and dose-dependent effects of apelin and cyclic analogues on phosphorylation of Akt and ERK in APJ/293-T cells. (A) Cells were exposed to apelin and cyclic analogues (at $1 \mu \mathrm{M}$ ) for indicated times. (B) Cells were exposed to apelin and cyclic analogues at indicated negative logarithm of concentration for 5 min. Cell lysates were blotted with anti-phosphorylated Akt (Ser473) and anti-phosphorylated ERK1/2 (Thr202/Tyr204) antibodies. The blots were stripped and reprobed with anti-Akt and anti-ERK2 antibodies. Reproducibility was observed in two independent experiments. None, unstimulated. 
A
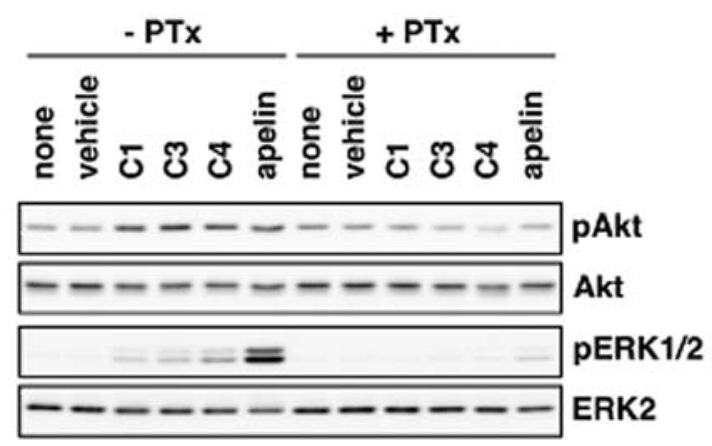

B
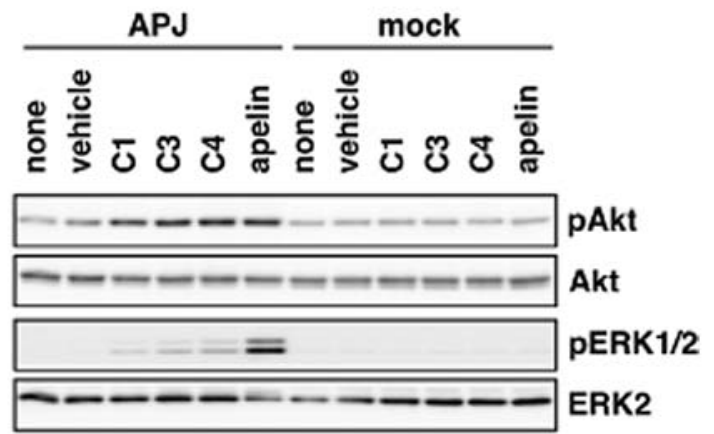

Figure 4. Effects of apelin and cyclic analogues on phosphorylation of Akt and ERK in PTx-treated APJ/293-T and mock/293-T cells. (A) APJ/293-T cells treated with PTx (right) or without (left) were exposed to apelin and cyclic analogues (at $1 \mu \mathrm{M}$ ) for $5 \mathrm{~min}$. (B) APJ/293-T (left) and mock/293-T (right) cells were exposed to apelin and cyclic analogues (at $1 \mu \mathrm{M}$ ) for $5 \mathrm{~min}$. Cell lysates were blotted with anti-phosphorylated Akt (Ser473) and anti-phosphorylated ERK1/2 (Thr202/Tyr204) antibodies. The blots were stripped and reprobed with anti-Akt and anti-ERK2 antibodies. Reproducibility was observed in two independent experiments. None, unstimulated.

of Akt was not changed at $1 \mathrm{nM}$ pE-apelin-13 compared with none and vehicle, but was increased to a similar extent in a concentration range from $10 \mathrm{nM}$ to $1 \mu \mathrm{M}$. In contrast, the phosphorylation levels of ERK1/2 were increased in a pEapelin-13 dose-dependent manner. Exposure to the cyclic analogues at high concentrations $(\mathrm{C} 1 ; 1 \mu \mathrm{M}, \mathrm{C} 3$ or $\mathrm{C} 4$; from $100 \mathrm{nM}$ to $1 \mu \mathrm{M}$ ) induced the increments of the phosphorylation levels of both Akt and ERK1/2 in a dosedependent manner, whereas at low concentrations, they did not increase. The induced phosphorylation of both Akt and ERK1/2 in APJ/293-T cells was almost abolished with PTx pretreatment (Fig. 4A), and no effects of any peptide (at $1 \mu \mathrm{M}$ ) were observed in the mock/293-T cells (Fig. 4B). These signaling assays demonstrated the potency of all cyclic analogues to activate both Akt and ERK1/2 in APJdependent and PTx-sensitive manner.

\section{Discussion}

APJ is thought to be a novel therapeutic target of cardiovascular dysfunction. The ameliorative effectiveness of apelin administration was demonstrated in pathological animal models, however, the effective dose was too high (12). Moreover, it is implied that exogenous apelin might be unstable and rapidly hydrolyzed by various peptidases including ACE2 (20). Therefore, it is necessary to modify apelin fragment to raise the efficacy as a drug and its physiological stability.

Several investigators have reported the structure-activity relationship (SAR) analysis of linear apelin analogues at human APJ. Medhurst et al (19) reported that 'RPRL' (ArgPro-Arg-Leu) motif in the amino-terminus of apelin-13 was an important motif for binding to APJ. On the other hand, Fan et al (21) demonstrated that the positive charge and hydrophobic residues of apelin-13, such as Arg and Pro, were very important for its functional potency and binding affinity. They also observed the secondary structure of apelin-36 and apelin-13 using Circular dichroism spectroscopy. Both peptides were shown to take unordered structure in aqueous solution. Despite these detailed analyses, there is no available pharmacophore model of apelin at APJ, because of its high dynamic conformational diversity. In this situation, one might expect that the cyclization, another SAR analysis method, is one of the most useful approaches to design drug-like apelin analogues.

Cyclization applies conformational restriction to a peptide, resulting in the improvement of its stability and bioactivity. There are several successful applications of cyclization to a peptidic ligand. For example, the bioactive cyclic analogues of somatostatin-derived peptides were synthesized $(22,23)$. The cyclic hexapeptide of somatostatin was shown to contain pharmacophore for somatostatin type 2 receptor (sst2), allowing the discovery of highly active pharmacological agents (15). It was also reported that the N-terminal cyclic motilin partial peptides were identified as neutral motilin antagonist (24), and that the bioactivity of the minimal histogranin tetrapeptide for dopamine $\mathrm{D}_{2}$ receptor was potentiated by amino acid replacement and cyclization (25). These studies showed that the cyclization could fix a peptide ligand in rigid conformation so as to activate or inactivate its specific receptor. Subsequent active core exploration will contribute to a pharmacophore search from non-peptidic small compound library. Furthermore, bioactive peptidic analogue will provide a therapeutic importance for the target receptor at an early stage in the drug development process. However, there is no report to apply cyclization to apelin yet.

In this study, we attempted to generate the cyclic apelin analogues. Both apelin-12 and pE-apelin-13 are thought to be the minimal active units of apelin. The amino-terminal pyroglutamyl residue in pE-apelin-13 is considered to contribute to its stability, but is not essential for its functional potency and binding at APJ $(3,26)$. Therefore, we selected the apelin-12 as a template for cyclic analogues, and assessed the effect of cyclization on apelin-12. We successfully synthesized three cyclic analogues of apelin-12 fragment and subsequently evaluated their bioactivities in a recombinant human APJexpressed cell line. In our cell-based assays, we used pEapelin-13 as a reference drug because of its well-established bioactivity in vitro assays $(16-19,21,26)$. From the cAMP assay, we identified the functional agonistic activities of all 
cyclic apelin analogues. Importantly, these analogues could induce the activation of Akt and ERK1/2 in PTX-sensitive manner.

The Akt and MAPK (mitogen-activated protein kinase) family are key molecules that mediate $G$ protein-coupled receptor-dependent signal transduction involved in the control of cell cycle progression, proliferation, migration, apoptosis and survival $(27,28)$. In various cell lines and primary neurons, it was shown that apelin-dependent activation of PI3K/Akt and MAPK signaling pathways resulted in cell proliferation, migration and survival $(17,18,29,30)$. Moreover, apelin was shown to produce direct cardioprotective actions against myocardial ischemia-reperfusion injury of mouse via the PI3K/Akt and MEK1/ERK1/2 signaling pathways (12). In this study, the activation of Akt and ERK1/2 induced by cyclic analogues suggested the potential activities of cyclic apelin analogues as a therapeutic agent in cardiovascular diseases as well as endogenous apelin.

In conclusion, by using cyclization methods, we established novel cyclic apelin analogues as the effective agonist for APJ. These cyclic apelin analogues may be useful for early validation of APJ and its target disease in a drug discovery. For this purpose, further evaluation will be needed on the stability and binding affinity of these peptides. The characterization of cyclic apelin analogues may provide insight into APJ-related cardiovascular diseases.

\section{Acknowledgements}

We acknowledge Akemi Ochiai, Kazuko Abe and Shina Seki for technical assistances. This work was supported by a Grant-in-Aid for Scientific Research (S) from the Ministry of Education, Culture, Sports, Science and Technology of Japan.

\section{References}

1. O'Dowd BF, Heiber M, Chan A, et al: A human gene that shows identity with the gene encoding the angiotensin receptor is located on chromosome 11. Gene 136: 355-360, 1993.

2. Tatemoto K, Hosoya M, Habata Y, et al: Isolation and characterization of a novel endogenous peptide ligand for the human APJ receptor. Biochem Biophys Res Commun 251: 471-476, 1998.

3. Zou MX, Liu HY, Haraguchi Y, Soda Y, Tatemoto K and Hoshino H: Apelin peptides block the entry of human immunodeficiency virus (HIV). FEBS Lett 473: 15-18, 2000.

4. Lee DK, George SR and O'Dowd BF: Unravelling the roles of the apelin system: prospective therapeutic applications in heart failure and obesity. Trends Pharmacol Sci 27: 190-194, 2006.

5. Japp AG and Newby DE: The apelin-APJ system in heart failure Pathophysiologic relevance and therapeutic potential. Biochem Pharmacol 75: 1882-1892, 2008.

6. Cayabyab M, Hinuma S, Farzan M, et al: Apelin, the natural ligand of the orphan seven-transmembrane receptor APJ, inhibits human immunodeficiency virus type 1 entry. J Virol 74: 11972-11976, 2000.

7. Szokodi I, Tavi P, Foldes G, et al: Apelin, the novel endogenous ligand of the orphan receptor APJ, regulates cardiac contractility. Circ Res 91: 434-440, 2002.

8. Berry MF, Pirolli TJ, Jayasankar V, Burdick J, Morine KJ, Gardner TJ and Woo YJ: Apelin has in vivo inotropic effects on normal and failing hearts. Circulation 110: II187-II193, 2004.
9. Ishida J, Hashimoto T, Hashimoto Y, et al: Regulatory roles for APJ, a seven-transmembrane receptor related to angiotensintype 1 receptor in blood pressure in vivo. J Biol Chem 279: 26274-26279, 2004

10. Ashley EA, Powers J, Chen M, et al: The endogenous peptide apelin potently improves cardiac contractility and reduces cardiac loading in vivo. Cardiovasc Res 65: 73-82, 2005.

11. Jia YX, Pan CS, Zhang J, et al: Apelin protects myocardial injury induced by isoproterenol in rats. Regul Pept 133: 147-154, 2006.

12. Simpkin JC, Yellon DM, Davidson SM, Lim SY, Wynne AM and Smith CC: Apelin-13 and apelin-36 exhibit direct cardioprotective activity against ischemia reperfusion injury. Basic Res Cardiol 102: 518-528, 2007.

13. Smith CC, Mocanu MM, Bowen J, et al: Temporal changes in myocardial salvage kinases during reperfusion following ischemia: studies involving the cardioprotective adipocytokine apelin. Cardiovasc Drugs Ther 21: 409-414, 2007.

14. Kleinz MJ and Baxter GF: Apelin reduces myocardial reperfusion injury independently of PI3K/Akt and P70S6 kinase. Regul Pept 146: 271-277, 2008.

15. Klabunde T and Hessler G: Drug design strategies for targeting G-protein-coupled receptors. Chembiochem 3: 928-944, 2002.

16. Masri B, Lahlou H, Mazarguil H, Knibiehler B and Audigier Y: Apelin (65-77) activates extracellular signal-regulated kinases via a PTX-sensitive G protein. Biochem Biophys Res Commun 290: 539-545, 2002.

17. Masri B, Morin N, Cornu M, Knibiehler B and Audigier Y: Apelin (65-77) activates p70 S6 kinase and is mitogenic for umbilical endothelial cells. FASEB J 18: 1909-1911, 2004.

18. Hashimoto $\mathrm{Y}$, Ishida J, Yamamoto R, et al: G protein-coupled APJ receptor signaling induces focal adhesion formation and cell motility. Int J Mol Med 16: 787-792, 2005.

19. Medhurst AD, Jennings CA, Robbins MJ, et al: Pharmacological and immunohistochemical characterization of the APJ receptor and its endogenous ligand apelin. J Neurochem 84: 1162-1172, 2003

20. Vickers C, Hales P, Kaushik V, et al: Hydrolysis of biological peptides by human angiotensin-converting enzyme-related carboxypeptidase. J Biol Chem 277: 14838-14843, 2002.

21. Fan X, Zhou N, Zhang X, et al: Structural and functional study of the apelin-13 peptide, an endogenous ligand of the HIV-1 coreceptor, APJ. Biochemistry 42: 10163-10168, 2003.

22. Veber DF, Holly FW, Paleveda WJ, et al: Conformationally restricted bicyclic analogs of somatostatin. Proc Natl Acad Sci USA 75: 2636-2640, 1978.

23. Rohrer SP, Birzin ET, Mosley RT, et al: Rapid identification of subtype-selective agonists of the somatostatin receptor through combinatorial chemistry. Science 282: 737-740, 1998.

24. Haramura M, Okamachi A, Tsuzuki K, et al: Design and synthesis of N-terminal cyclic motilin partial peptides: a novel pure motilin antagonist. Chem Pharm Bull (Tokyo) 49: 40-43, 2001.

25. Le HT, Lemaire IB, Gilbert AK, Jolicoeur F and Lemaire S: Bioactive peptidic analogues and cyclostereoisomers of the minimal antinociceptive histogranin fragment-(7-10). J Med Chem 46: 3094-3101, 2003.

26. El Messari S, Iturrioz X, Fassot C, De Mota N, Roesch D and Llorens-Cortes C: Functional dissociation of apelin receptor signaling and endocytosis: implications for the effects of apelin on arterial blood pressure. J Neurochem 90: 1290-1301, 2004.

27. New DC, Wu K, Kwok AW and Wong YH: G protein-coupled receptor-induced Akt activity in cellular proliferation and apoptosis. FEBS J 274: 6025-6036, 2007.

28. Rozengurt E: Mitogenic signaling pathways induced by $G$ protein-coupled receptors. J Cell Physiol 213: 589-602, 2007.

29. Tang SY, Xie H, Yuan LQ, et al: Apelin stimulates proliferation and suppresses apoptosis of mouse osteoblastic cell line MC3T3-E1 via JNK and PI3-K/Akt signaling pathways. Peptides 28: 708-718, 2007.

30. O'Donnell LA, Agrawal A, Sabnekar P, Dichter MA, Lynch DR and Kolson DL: Apelin, an endogenous neuronal peptide, protects hippocampal neurons against excitotoxic injury. J Neurochem 102: 1905-1917, 2007. 